\title{
IoT based Smart Vehicle Monitoring System : A Systematic Literature Review
}

\author{
Tanvir Rahman \\ Department of computer Science and Engineering (CSE), Stamford University, Bangladesh, Dhaka, Bangladesh \\ email : next.innovation98@gmail.com
}

\begin{abstract}
Article Info

Volume 8 Issue 1

Page Number: 12-20

Publication Issue :

January-February-2021

Article History

Accepted : 01 Jan 2021

Published : 10 Jan 2021

This paper provides a complete over view of the current research state of Smart vehicle tracking System with GPS and cellular network. This paper consists of several review aiming to reveal the relevance and methodologies of this research area and create a foundation for future work. In this paper an advanced vehicle observation and IOT based tracking system and autopilot navigation system based on Machine Learning and neural Networking is proposed with all possible scientific validations of the model. The primary purpose of monitoring the vehicles which are moving from one place to the other in order to provide better A.I based autopilot navigation system, safety and security. The proposed method Combined the idea of Java programming, Neural networking concept with machine learning capability processing data with MediaTek mobile processor and its sophisticated features of storing data into several databases. Google Map Engine API v3 was used to display and sense the graphical images of the map and a Vision recognition server system is used to compare and represent the map API in a more realistic look. The proposed project includes the implementation of Global Positioning System (GPS), GPRS and GSM technology for vehicle tracking and monitoring on real time basic purpose using SIM module.[3] The GPS receiver installed o tracking device provides real-time Geolocation Co-ordinate of site of the vehicle; 3 adjacent GSM cellphone tower stationswill continuously broadcast co-ordinate of locations and the GPRS technology with TCP based protocol sends the tracking information to the central Monitoring and Imaging server which consist of 3 child servers i)data processing sever, ii) Image and vision based server and iii)A.I. based machine learning server calculate data and minimize the information and maps with the help of Google map API and thus an decision message for next Move/driving path isgenerated and transmitted to Smart Controlling Device to execute the instructions and to display it in the Monitor of car display and Integrated logged-IN andriod based Google Map
\end{abstract}


API version 3 app on real time basic. Hence, this system will monitor all the driving steps of the driver and provide the real time driving suggestions and feedback to the driver to ensure smooth and safe driving experience. The sensors like temperature sensor, altitude sensor and smoke sensor send data to the neural processing Server which diagnoses the health and safety measures of the vehicles and generates a report on Car display and andriod App interface if any risk issue is found by sensors.

Keywords : GPS, Autopilot system, GPRS, Machine learning, Safe-driving, wireless communication

\section{INTRODUCTION}

Global positioning System (GPS) has become the important part of modern Communication and vehicle tracking system. A GPS trackable chip is a device that receives signal from "Glonass satellite"[3][1] signal to determine the global positioning system to identify the correct location of a person, vehicle etc. The signal is sensed within a time intervals and data are stored and processed in a microchip.[11] An andriod OS operated device with Intelligent Google map API, a GPS signal receiver Antenna[8], A GPRS and GSM receiver Modem and Some Essential Sensors should be embedded to tracking device. User will interact with the system with a custom made Google Map enabled UserInterface with touch enabled display. The microprocessor chip is programmed to process the data and store Co-ordinate of geolocation or it may be transmitted via using TCP protocol to a central database Server and Google Map API, via GPRS packet transmit Antenna internet- connect device and GSM network with the GSM network support of 3 adjacent BTS[9] are also used to crossvalidate the acquired geolocation coordinate(current position) gained through the GPS signal receiver. Future self-operating/ self-driving automobile driving system is largely dependent upon the GPS technology for acquiring it's current location and adjacent location point. GPS system is based on a satellite-based radio navigation which continuously broadcast the system geolocation and time information to a GPS receiver anywhere on or near the Earth where there is an unobstructed line of sight to four or more GPS satellites.[1] The Central Data processing and Computational Sever is enriched with the Machine Learning[1] and neural networking algorithm to generate the next step move for the tracking enable vehicle with the implementation of artificial intelligence and some Sensors installed in the vehicles to sense and collect data about speed, adjacent points, adjacent obstacles and possible turn-out points for vehicles mobility in the road. In this process Model Initially, the GPS continuously takes input data from the satellite and stores the latitude and longitude values in microcontroller's buffer.[3][5] If we have to track the vehicle, [5][3]we need to send a message to GSM device, by which it gets activated. It also gets activated by detecting accident on the IR sensor, by detecting fire on the temperature sensor, by detecting theft connected to vehicle. [1][2]. Parallelly deactivates GPS with the help of relay .Once GSM gets activated it takes the last received latitude and longitude positions values from the buffer and sends a message to the particular number or laptop which is predefined in the program. Once message has been sent to the predefined device the 
GSM gets deactivated and GPS gets activated. That's why, GPS[1]system is the most convenient for today's global vehicle tracking and automotive self-operating vehicle system. This new technology, popularly called vehicle Tracking Systems which created many wonders in the security of the vehicle This hardware is fitted on to the vehicle in such a manner that it is not visible to anyone who is inside or outside of the vehicle. .[3][4]. Thus it is used as a covert unit which continuously or by any interrupt to the system, sends the location data to the monitoring unit. When the vehicle is stolen, the location data from tracking system can be used to find the location and can be informed to police for further action. Some Vehicle tracking System can even detect unauthorized movements of the vehicle and then alert the owner.[9][1] This gives an edge over other pieces of technology for the same purpose This accident alert system in it detects the accident and the location of the accident occurred and sends GPS coordinates to the specified mobile, computer etc. The fire detector circuit in it is used to detect fire in the vehicle, if the temperature inside the vehicle goes above a certain limit then a warning.[3][4].By implementing the Certain technology, a vehicle tracking system can be the most efficient ,trustworthy and useful medium of day to day use for vehicle tracking and traffic monitoring.[5][9]

\section{RESEARCH METHODOLOGY}

To write a review about this certain Topic 20 individual research papers, journals on Auto-pilot navigation system, Machine learning ,3D mapping and tracking system based on GPS,GPRS modules were selected carefully to collect previous research contributions of various scientists. The idea of systematic literature review is a well-established approach to identify, examine, recheck and evaluate of all relevant topics and particular research question of interest [11]. Several case studies are likely to appear in formal research venues, as journals and professional conferences, rather than popular works.[16]. In doing case study research, the "case" being studied may be an individual, organization, event, or action, existing in a specific time and place. For instance, clinical science has produced both well-known case studies of individuals and also case studies of clinical practices. [15][16]. Research questions and methodologies were set according to the requirements and preferences of project pattern and working strategy.[3]A solid strategy was chosen to get a fair, and unbiased evaluation and modulation of approaches for handling variability in Smart Vehicle Tracking System using GPS and wireless communication network.[10][11].

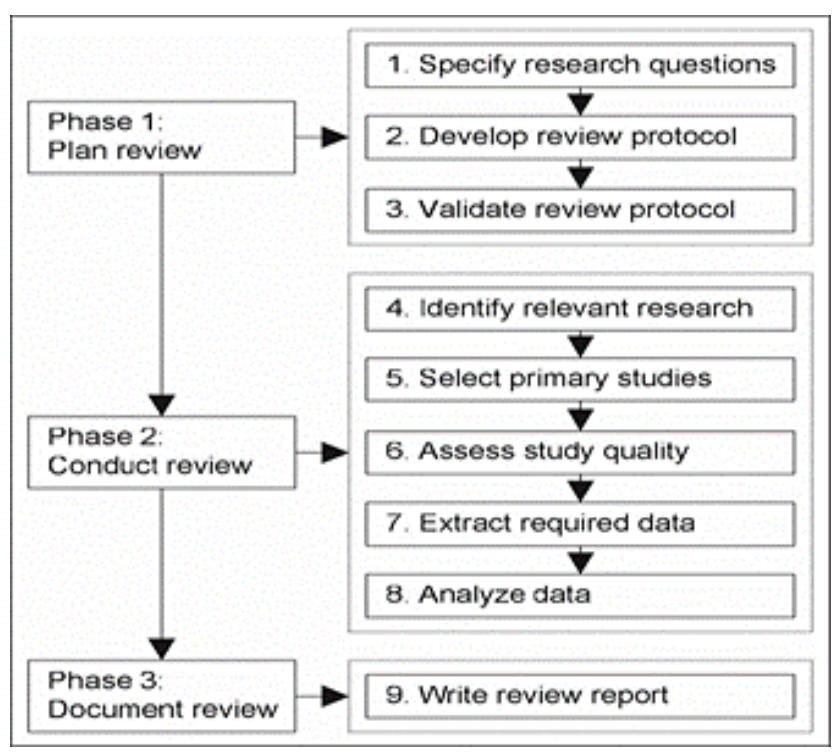

Figure 1 : This Figure of SLR process in this diagram was adapted from reference paper [11].

In this paper certain strategies were introduced to reduce the Chance of biasness and favourable expected output so research topic. Some certain were proposed to validate the accuracy of data source. Pilot search strategy was followed in this project. Based on the research questions identified and marked in this paper[3][1] , it was identified what data elements to extract from each and every individual study found in the search strategy.[2][3] More than 3 independent researchers and expert analysists were interviewed and more than 19 national and international 
published journal papers were reviewed and used for the primary data source of the proposed model. The effectiveness of the proposed model was crosschecked and scientifically proved by the different[9][14] validation techniques used by moderators and Editorial Team. Research questions were identified based on the real-life problem-based scenario, working challenges, technical limitations and based on the requirements and purpose of the project. [7][5][2]

\section{RESEARCH QUESTIONS}

"The primary target of this research is to investigate the extent to which the three dimensions of vehicle Tracking and Self navigating vehicle monitoring System projects" [1][10].Global positioning system based on geo-location Satellites, Vehicle tracking and monitoring strategy for auto pilot navigation system and the security and the integrity measurement of the vehicle tracking system [4][5][11].To make this this eco-system user friendly, more convenient and compact the certain points of questions should be answered to maximize the cost efficiency, effectiveness and integrity of this system. Under the Criteria of the discussion, it was focused that the study examines the following set of research questions.[5]

RQ1: What are the vital components and Engineering strategies needed to develop the compact GPS based navigation system and how to calculate the geolocation measurement accurate for location-based service?[10][3]

RQ2: What correlations can be established between the geo-location tracking, autopilot navigation system, User-interface of $3 \mathrm{~d}$ mapping system and the GPRS data sync between the devices installed and central data processing station their evaluation criterion? e.g., working strategy , long term sustainability, maintainability and quality?

RQ3 : Does this technology is efficient and cost friendly to implement it in a tradition civilian vehicle and does this technology easily operate-able by using a User Interface?

RQ4: What kind of metrics and collaboration models can be defined for modeling and generalizing the coevolution of sustainable vehicle tracking and auto pilot vehicles?[6]

RQ5 : What are the main factors, Components ,Sensors needed to implement an interactive System which will interact both with the GPRS data and Global positioning System to sensing and obtaining real time location and to find the safest collision free route to move on the correct direction? RQ6: How to analyse, the safety measurements ,security and the integrity of this geolocation-based navigation system and what are the security standard old this proposed navigation system ? [5]

RQ7:What types of data encryption and data security algorithms are used to secure the data communication path between the Tracker (Smart I-device) and the central server Station ?[11][7]

RQ8:Does the proposed model can Replace the Manual driving system with an efficient fully automated self-driving car system?[8][9]

RQ9:Does this proposed model of autopilot driving and self-navigation system has any alternative communication medium or technology to work on the most extreme conditions and in the time of natural disaster?[15]

RQ10 : Doesthe Systemhas any learning ability based on prior driving experience and what is the effectiveness of Autopilot navigation system ?[1][2]

R11:Does this proposed model of autopilot driving and self-navigation system is applicable for a less/weak internet infrastructure equipped country ?[4]

R12:Does this proposed model of autopilot driving and self-navigation system has any alternative running strategy hasany backup plan to resolve any Computational or data deficiency problem?

R13:Does this proposed model of autopilot driving and self-navigation system has any alternative communication medium or technology to work on 
the most extreme conditions and in the time of natural disaster?

\section{EXISTING RELATED WORK}

This certain literature review about the previous work related to existing vehicle tracking system [7] focused only tracking of the vehicle but there was a great lack of auto-pilot navigation system and security measures to prevent accidents.[6]

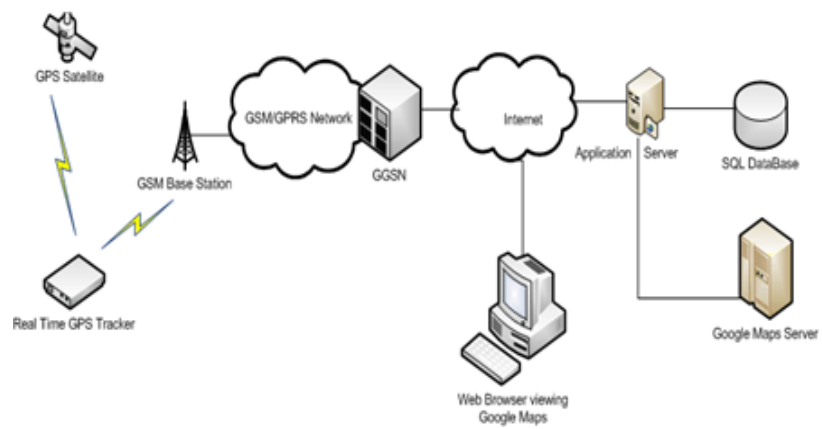

Figure 2 : Vehicle tracking System based on Google API collected from the international Paper [1]

Working procedure Flowchart is also included in reference Paper[10]. In this proposed model the researcher implemented a mobile packet data based real-time GPS tracker with integrated Google Maps API V3. In this project, they used the GPS receiver chip to receive real-time geolocation co-ordinate information and data is transferred over a GPRS link with latest encapsulation and TCP based secure gateway of wireless cellular mobile operators to a remote Data processing server using cellular GPRS packet.[7][6][11] The Data processing server stores the incoming co-ordinate data and match the coordinate data information with the co-ordinate lookup table. . Then all the data of geolocation coordinate integrates this information into Google Maps cloud serve through Google Maps API V3 which then reply a 2D based Map interface to the Central data processing Server and then the central server again send the 2D map interface and co-ordinate data information to the tracking device's display monitor installed in the vehicle . [11]. When a user with a username and password click on the specific tracking page, the page serves up an HTML and PHP based responsive webpage with an embedded JavaScript technology.[7][8]The user-end web browser uses JavaScript request at every single second and retrieve every single data/ mapping interface passed by XML file from the central data processing server. As the real time geo-location co-ordinate and 2D mapping diagram[7] is retrieved every second and the maps reupdated at every single second, a real time basic vehicle tracking system is achieved.[10][11].

\section{LIMITATIONS OF EXISTING MODEL}

The existing reviewed model had Lower accuracy and Lack of artificial intelligence \& self-driving autopilot monitoring system. There was a great lack of data collecting sensors like motion sensor, heat sensor, altitude sensors etc. There was not any data encapsulation and protocol model. The Lack of controlling mobile application is a serious obstacle. The existing models were not totally dependable and there were a lot of security and safety vulnerability issues.

\section{PROPOSED SYSTEM}

In this proposed model, GPS (Global Positioning System), GPRS (General packet Radio Services),GSM technology, Artificial Intelligence with Neural networking and Machine Learning capability and Cloud Based google Map Server are used to run this complex real time auto-pilot navigation system. A GPS signal receiver and GPRS, GSM and wireless radio band modem with SIM-900 slot with a Snapdragon 845 mobile processor chip ,DDR4 Ram, Flash storage and integrated (GSM/GPRS/LTE) signal broadcasting antennas are combined and integrated in a single package device named "I-Tracker" which is manufactured by a Third-party organization and it will be placed inside any Vehicle.

"GPS has provided positioning, navigation, and timing services to military and civilian users on a continuous worldwide basis since first launch in 1978". [10][1] By 
using the GPS technology, An unlimited number of users can accurately receive GPS signal with a GPS receiver which can determine accurate time and location, in any weather, day or night, anywhere in the world at real time basic.[3][7].
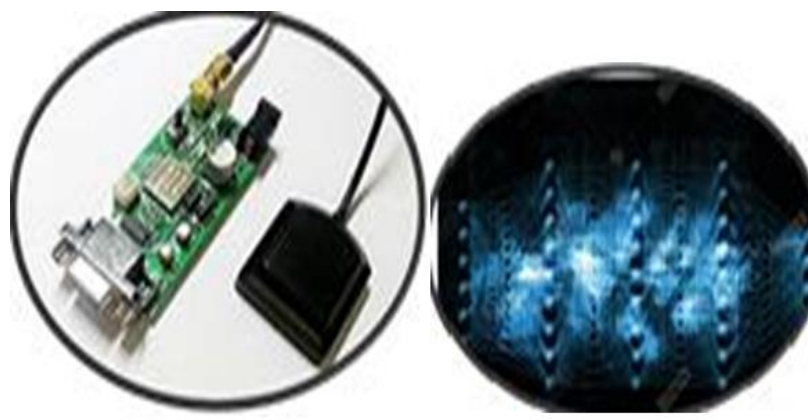

Figure 3 : GPSmodule [5] Figure 4 : Artificial Neural Networking

"Artificial neural networks or connectionist systems are computing systems vaguely inspired by the biological neural networks that constitute animal brains which has the Machine learning Capability and can produce any real-time Decisions based on previous learning"

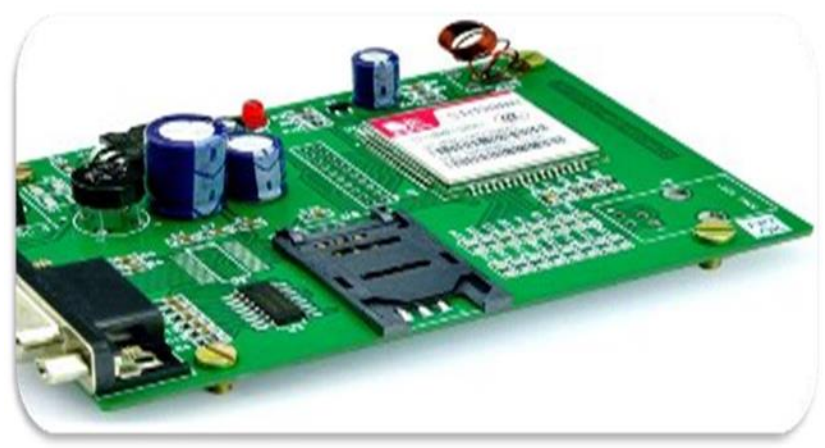

Figure 4 : GSM,GPRS, Modem, antenna and processor integrated chip [5][10]

“GPRS stands for General Packet Radio Service. GPRS is packet-switched wireless medium radio communication service which provides data rates ranging from 56 to $114 \mathrm{kbps.”} \mathrm{[13][10].}$

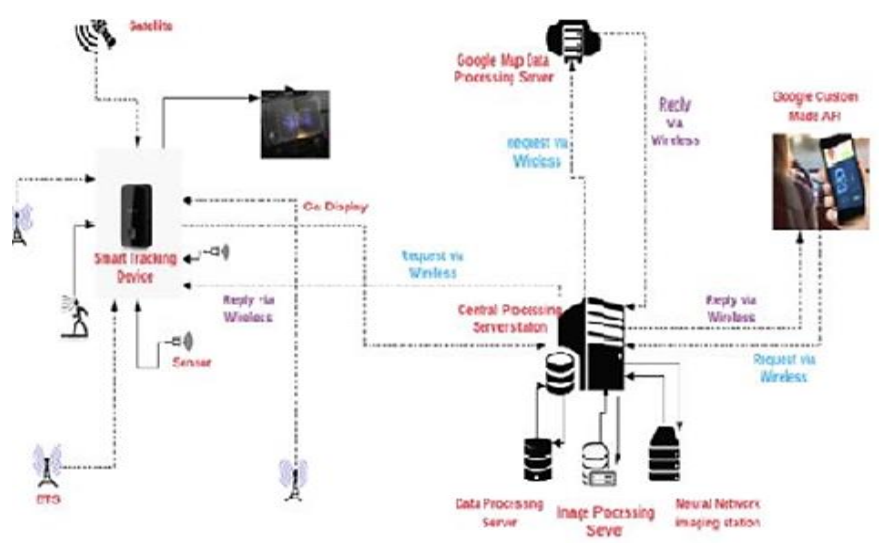

Figure 6 : Our Newly designed Proposed model Working procedure VIII. WORKING PROCEDURE

In the proposed model, a real time artificial intelligence-based mobile GPS tracker with integrated Google Maps API V4 has been implemented with Real time auto-pilot navigation system run on several machine learning algorithms and vision and image processing unit. GPSsignal receiver antenna senses and receives the real time geo-location co-ordinate information are sent by the 'Glonass' Satellite systemin every single moments. Three nearest dedicated BTS stations continuously send geo-location coordinate information via GSM gateway which geo-locational co-ordinate information are also used to validate and crossvalidate/check the geolocations-ordinates gained from GPS tracking Module at real time base. The tracking device "I-Tracker" includes integrated GPRS and GSM modem, Signal antenna broadcaster with a lot of sensors for collecting information like speed, temperature, altitude, surrounding obstacles and smoke detection data. The all gathered data on real time basic like reporting sensors collected data, Geolocationco-ordinate data are being highly synthesized and analysed by the 'Snapdragon 845 'processor chip and then all data will be encapsulated in binary combination and placed in a object of data set. Then all this object of real-time huge object dataset transferred over a GPRS link/LTE wireless medium by the Roaming GPRS broadcasting Antenna installed in our tracking device via mobile operator's GGSN to the "Central data processing server" overaTCP connection with latest data security and encryption technique. 
"The central processing Server station" embedded with 3 different purposes servers i)Data -Processing server, ii)Vision and Image Processing server and iii) Neural networking-based Decision generator Server which are responsible for calculating and analysing data, Image processing Server and Neural networking and imaging server Unit. The central processing

Server station stores the incoming positional data. The central processing Server station transfers the data of the global positioning co-ordinate basepoints and geo-location information to the cloud based central "Google map Server" with a TCP based connection inversing 0.10 second with the AJAX, JSON webservices technology for real time HTTP Request and Reply method. Google map server also reply the "central processing Server station" with the "3D based mapping Interface" and Mapping base-points. Then the "3D mapping Interface" is sent to the "Image processing and vision" server which're-visualizes the mapping dataad images and process the data to make the mapping interface into more realistic view and then "3D Mapping street view" with street navigation is sent back to the central processing Server station. At the same time, The neural processing Server analyses all supplied dataset and test cases and make a gamete of location information based on prior learning with the help of various machine learning algorithms and feedback. Then the neural processing server sends back a reply with the best possible move, next Safest path directory, possible risk evaluation and suggest best route to avoid collision with nearest moving object in the road etc decision making data to the connected "central processing Server station". Then ,all the processed data and driving instructions are placed in another object in encrypted format and then send back the processed data to the "I-Tracker" device which is installed in the vehicle over a TCP connection with latest data security and encryption techniques. After all, "I-Tracker" device installed in vehicle continuously senses and receives processed data from "Central processing server station" at the interval of every 0.10 second. Then the processed data/information are shown in Car/Vehicle display monitor and the suggested driving route and best optimal driving path are recommended to the drivers by voice navigation instruction. Every driving information /best driving routes are also broadcasted from the "central server station" for every individual connected vehicle with a unique broadcasting Identification number (VID).A dedicated custom made android application $\mathrm{d}$ is used to show all driving information, instructions, vehicle health diagnosis report and disaster alert notifications. A registered user with (VID ) number and password can login to the application interface. This App is used to connect with central processing Server to display tracking data from anywhere from the world for a specific trackable vehicle.

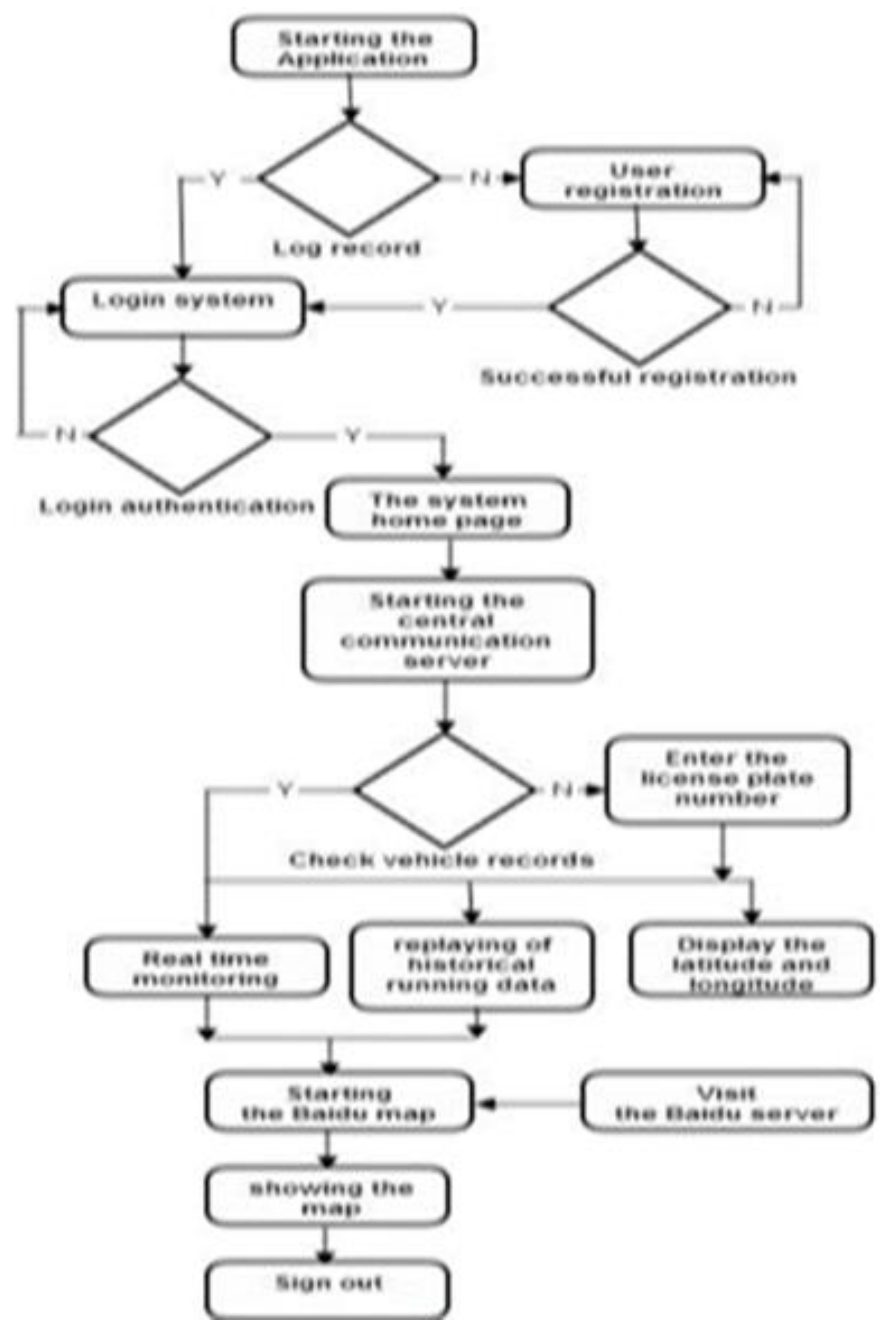

Figure 7 : System working principle in flowchart, (idea and primary information is collected from Reference paper [10]) 


\section{EQUATIONS}

All the necessary and relevant Mathematical equations and machine Learning algorithms derivations are available in Reference paper [1] from (4-8) page.

\section{RESULTS}

Table.1: Evolution Tracking of Vehicle

\begin{tabular}{|c|c|c|c|}
\hline Parameter & Suggested Range & Value above & Status \\
\hline $\begin{array}{l}\text { Near by } \\
\text { still/moving } \\
\text { object } \\
\text { Detection }\end{array}$ & $\begin{array}{l}\text { At least } 2 \text { feet } \\
\text { distance range from } \\
\text { the running vehicle }\end{array}$ & $\begin{array}{l}1 \text { feet range } \\
\text { from the run }\end{array}$ & $\begin{array}{l}\text { Slowing down } \\
\text { speed of vehicle } \\
\text { and generate } \\
\text { alarm }\end{array}$ \\
\hline $\begin{array}{l}\text { Human } \\
\text { Scanner\& } \\
\text { Driving } \\
\text { angle } \\
\text { analyzer }\end{array}$ & $\begin{array}{l}\text { Detect Human } \\
\text { position and } \\
\text { recalculate driving } \\
\text { angle to avoid } \\
\text { collision } \\
\text { Before } 1.5 \text { feet } \\
\text { distance }\end{array}$ & $\begin{array}{l}\text { Detect Human } \\
\text { position and } \\
\text { recalculate } \\
\text { driving angle to } \\
\text { avoid collision } \\
\text { Before } 0.5 \text { feet } \\
\text { distance }\end{array}$ & $\begin{array}{l}\text { Immediately } \\
\text { slowing vehicle } \\
\text { to stop vehicle } \\
\text { within less than } \\
2 \text { seconds and } \\
\text { Generate high } \\
\text { buzzer alarm }\end{array}$ \\
\hline $\begin{array}{l}\text { Over Speed } \\
\text { or High } \\
\text { altitude } \\
\text { driving from } \\
\text { sea level }\end{array}$ & $\begin{array}{l}80-90 \mathrm{~km} / \mathrm{h} \text { based on } \\
\text { traffic-condition } \\
\text { (Highway), } \\
20-40 \mathrm{~km} / \mathrm{h} \text { crowded } \\
\text { road or near school, } \\
\text { hospital }\end{array}$ & $\begin{array}{l}95-120 \mathrm{~km} / \mathrm{h} \\
\text { based on road } \\
\text { traffic } \\
\text { condition }\end{array}$ & $\begin{array}{l}\text { Slowing down } \\
\text { speed of vehicle } \\
\text { and generate } \\
\text { alarm }\end{array}$ \\
\hline Blink of eye & 1-3 Blinks / 5 sec. & $\begin{array}{l}>3 \text { Blinks } \\
\text { / } 5 \mathrm{sec}\end{array}$ & generate alarm \\
\hline $\begin{array}{l}\text { Unguided or } \\
\text { wrong } \\
\text { directional } \\
\text { move }\end{array}$ & 2 wrong turn /move & $\begin{array}{l}3 \text { wrong turn } \\
\text { /move }\end{array}$ & $\begin{array}{l}\text { Generate voice } \\
\text { alarm }\end{array}$ \\
\hline Temperature & $\begin{array}{l}\text { Up to } 50 \text { degree } \\
\text { congregate }\end{array}$ & $\begin{array}{l}>60 \text { degree } \\
\text { Centigrade }\end{array}$ & $\begin{array}{l}\text { Alert Driver by } \\
\text { Alarming }\end{array}$ \\
\hline
\end{tabular}

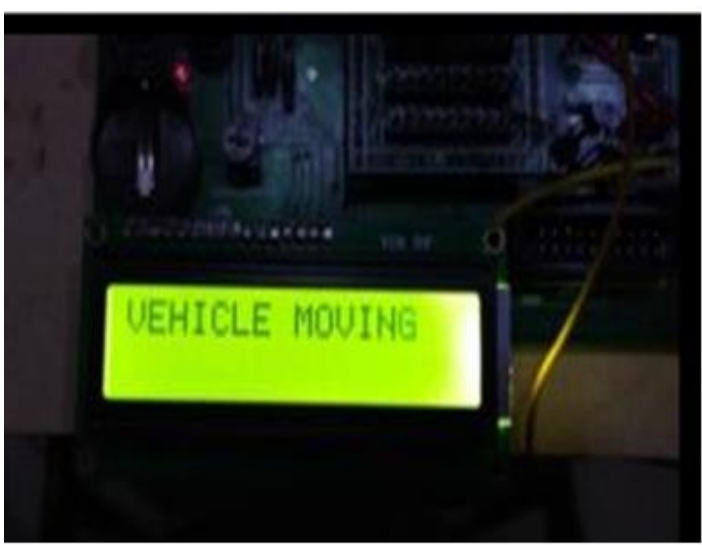

Figure 8 : showing vehicle movement

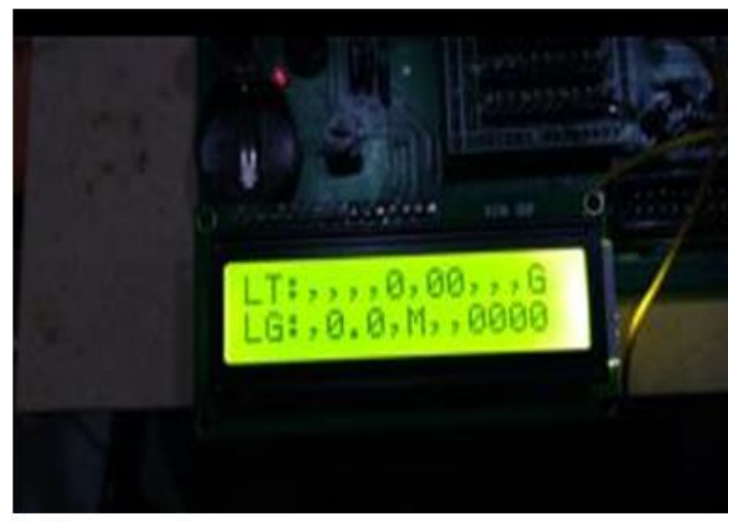

Figure 9 : showing geo-location

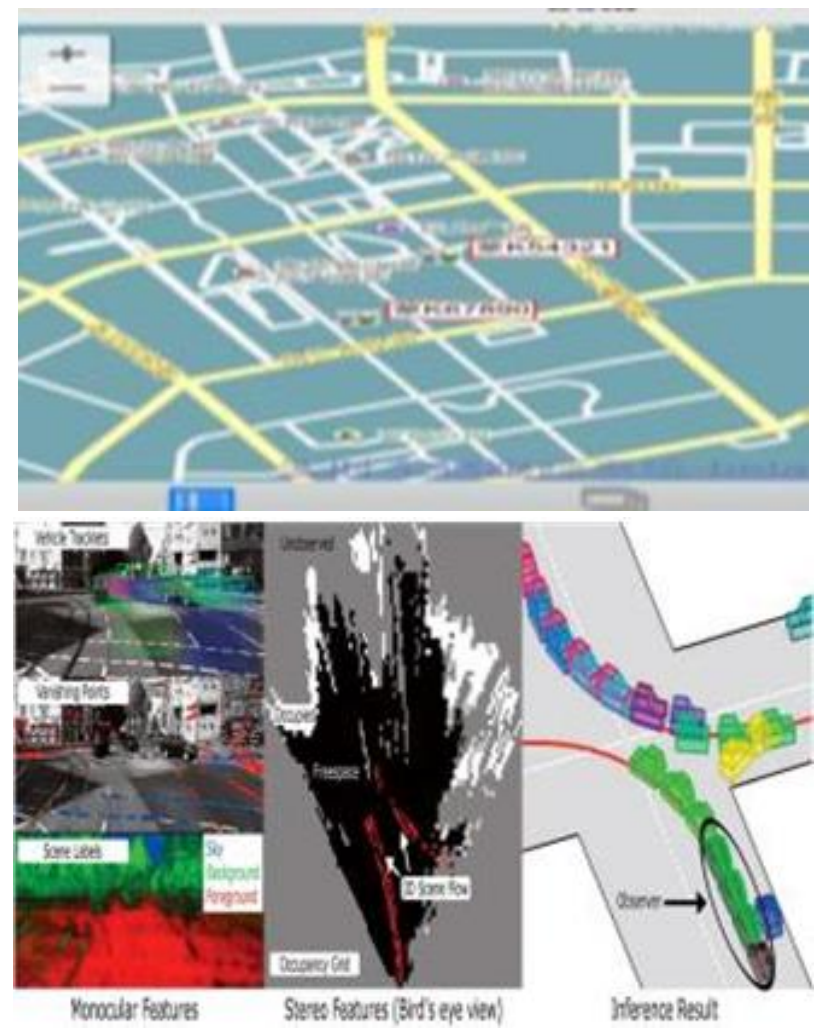

Figure 10 : Controlling mobile App \& Driving navigation

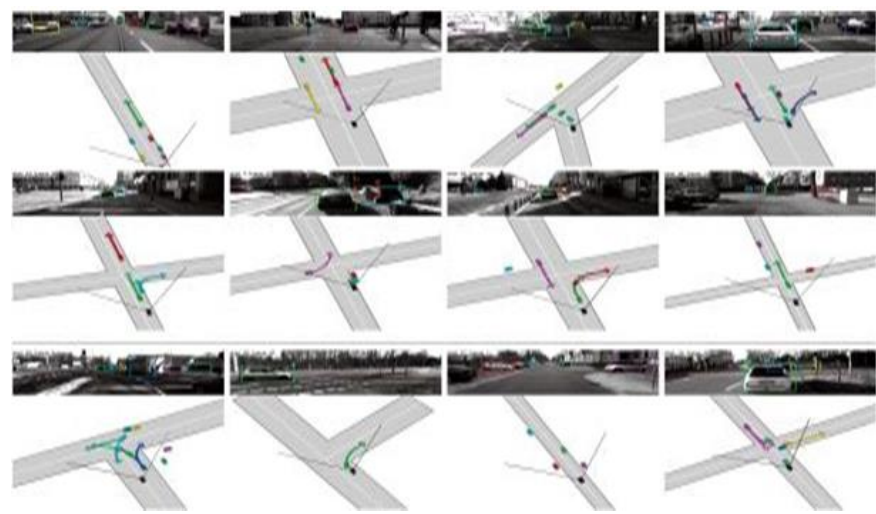

Figure $11: 3 \mathrm{~d}$ image scene of road (all Figures are collected from paper[2][10][11][12][3]) 


\section{VALIDATION}

This proposed model is designed based on the prior experiences of vehicle tracking system and auto-pilot driving system. The idea of Self driving auto-pilot navigation and monitoring system has already been implemented by UBER, TESLA ,Google (fig:8,9,10) and a lot of mathematical derivations and proposed published model has validated the probability of high success rate for implementing a smart self-driving car concept .Our proposed model was tested and validated by using a scientific formulas and more than 3 individual experts relevant to the field have secured the validity and effectiveness of our proposed model. More than 20 scientific journals on Autopilot navigation system were reviewed to gather scientific evolution and to know the working principle of selfdriving car.

\section{CONCLUSION}

In this review paper,A new strategy of Smart Self driving auto-pilot vehicle monitoring system is introduced by using GPS tracking of vehicles, GPRS,LTE,GSM technology and Machine learning algorithms. Though the proposed model is under testing phase, but in near future the system highlights the improvements in end user experience and it will help to reduce the road accident rate and will improve the road safety standard. With the help of artificial intelligence Machine learning technology will significantly improve the user/drivers driving experience on the road. This developed model is fully secured based on latest data encryption algorithms which allows a fair access to all users. Various Sensors installed in the vehicle continuously sense and collect important data at every single second to diageneses the vehicle health and safety measure on real time basics and alert and notification-based service is highly helpful to user for better driving experience and extended security measures. This proposed model can be provided as a premium and Loyalty service to consumer, which will help it, make sustainable. As It is an open-source Project, the developed models and source code will allow developers to build further on this project by predicting highly localized observational parameters like environmental pollution, local weather and humidity point, etc.[4][5]. Finally, this proposed model is a best alternative solution to traditional manual driving system.

Hopefully in near future it will change / replace the whole concept of traditional diving policy by fully automated self-driving vehicle concept which will significantly improve the road security and safety standard.

\section{Acknowledgment}

This Systematic literature Review is fully supervised by DR. M. M. MAHBUBUL SYEED, Head of Department, Faculty of Computer Science, AIUB.. For the supreme contribution of this paper are dept to our honorable supervisor .. Finally, we want to thank to our family members and friends for their extreme supports and inspiration.

\section{XII.REFERENCES}

[1]. MihirGarude, NirmalHaldikar (September 2014) Real Time Position Tracking System Using GoogleMaps APIV3, Volume4, Issue9, edn., International Journal of Scientific and Research Publications:

[2]. A.Anusha,Syed Musthak Ahmed (2017)VEHICLE TRACKINGAND MONITORING SYSTEM TO ENHANCE THE SAFETY AND SECURITY DRIVING USING IoT, edn., 2017 International Conference on Recent Trends in Electrical, Electronics and Computing Technologies: .

[3]. Andreas Geiger, Martin Lauer, Christian Wojek, Christoph Stiller, and Raquel Urtasun (2014) 3D Traffic Scene Understanding from MovablePlatforms, VOL. 36, NO. 5, MAY edn., 
IEEE TRANSACTIONS ON PATTERN ANALYSIS AND MACHINE INTELLIGENCE: .

[4]. C. Andrieu, N. de Freitas, A. Doucet, andM. I. Jordan, "An introductionto MCMC for machine learning," Mach. Learn., vol. 50, no. 1-2, pp. 543, 2003.

[5]. M. Andriluka, S. Roth, and B. Schiele, "Monocular 3D pose estimation and tracking by detection," in Proc. CVPR, San Francisco, CA, USA, 2010.

[6]. S.Bao,M.Sun,and S.Savarese, "Toward coherent object detection and scene layout understanding," in Proc. CVPR, San Francisco, CA, USA, 2010.

[7]. O. Barinova, V. Lempitsky, E. Tretyak, and P. Kohli, "Geometric image parsing in man-made environments," in Proc. ECCV, Berlin, Germany, 2010.

[8]. C. M. Bishop, Pattern Recognition and Machine Learning, 1st ed. New York, NY, USA: Springer, 2006.

[9]. M. D. Breitenstein, F. Reichlin, B. Leibe, E. Koller-Meier, and L. Van Gool, "Robust tracking-by-detection using a detector confidence particle filter," in Proc. ICCV, Kyoto, Japan, 2009. Autonomous Challenge," Annu. Rev. Control, vol. 36, no.

[10]. A.Geiger,P.

Lenz,andR.Urtasun, "Arewereadyforautonomou s driving? The KITTI vision benchmark suite," in Proc. CVPR,Providence, RI, USA, 201

[11]. Matthias Galster,Matthias Galster, Danny Weyns,Dan Tofan,BartoMichalik, and Paris Avgeri(MARCH 2014) Variability in Software Systems- A Systematic Literature Review, VOL. 40

[12]. A. Geiger, M. Roser, and R. Urtasun, "Efficient large-scale stereo matching," in Proc. ACCV, Queenstown, New Zealand, 2010.

[13]. A. Geiger, C. Wojek, and R. Urtasun, "Joint 3D estimation of objects and scene layout," in Proc. NIPS, 2011.
[14]. A. Gupta, A. A. Efros, and M. Hebert, "Blocks world revisited:

[15]. A. Ess, B. Leibe, K. Schindler, and L. V. Gool, "Robust multi-person tracking from a mobile platform," IEEE Trans. Team members contribution Pattern Anal. Mach. Intell., vol. 31, no. 10, pp. 1831-1846, Oct.

[16]. G. Hinton, "Training products of experts by minimizing contrastivedivergence," Neural Comput., vol. 14, no. 8, pp. 1771-1800 TransPattern Anal. Mach. Intell., vol. 31, no. 12, pp. 2196-2210, Dec. 2009.,

[17]. Parvez, M.Z.Ahmed, Rahman, "A theoretical model of GSM network based vehicle tracking system", Electrical and Computer Engineering.

[18]. Bing-fei Wu, Ying-Han Chen "An efficient Web-Based Tracking System through reduction of Redundant Connection"

\section{Cite this article as :}

Tanvir Rahman, " IoT based Smart Vehicle Monitoring System : A Systematic Literature Review, International Journal of Scientific Research in Science, Engineering and Technology(IJSRSET), Print ISSN : 2395-1990, Online ISSN : 2394-4099, Volume 8, Issue 1, pp.12-20, January-February-2021. Available at doi : https://doi.org/10.32628/IJSRSET207647 Journal URL : http://ijsrset.com/IJSRSET207647 